\title{
Fatores associados à depressão em pacientes oncológicos durante quimioterapia*
}

\author{
Factors associated with depression in cancer patients during chemotherapy
}

Taciana Cunha Arantes ${ }^{1}$, Vitória Eugênia Martins ${ }^{2}$, Amanda Silva Mendes $^{2}$, Andréa Mara Bernardes da Silva ${ }^{3}$, Adriana Cristina Nicolussi ${ }^{2}$

Objetivo: analisar os fatores associados à depressão, em pacientes oncológicos, durante quimioterapia. Métodos: estudo descritivo, transversal, realizado com 208 pacientes, durante quimioterapia. Utilizou-se de questionário para caracterização geral e Inventário de Depressão de Beck. Resultados: maioria era mulher, entre 40 e 79 anos, casadas, católicas, aposentadas/donas de casa, com ensino fundamental e encontrava-se sem depressão (71,2\%); enquanto 17,3\% apresentaram depressão e 11,5\% disforia. Nas associações, encontrouse significância estatística nas associações do Inventário de Depressão de Beck com realização de cirurgia $(\mathrm{p}=0,002)$, tempo decorrido desde cirurgia $(\mathrm{p}=0,014)$, tempo desde início da quimioterapia $(\mathrm{p}=0,030)$ e efeitos colaterais da quimioterapia $(\mathrm{p}=0,019)$. Conclusão: houve baixa incidência de depressão, contudo a frequência foi maior nos pacientes que não realizaram cirurgia concomitante, que tiveram quimioterapia iniciada até seis meses e que relataram mais efeitos colaterais durante o tratamento.

Descritores: Neoplasias; Depressão; Tratamento Farmacológico; Enfermagem Oncológica.

Objective: to analyze the factors associated with depression in cancer patients during chemotherapy. Methods: a descriptive cross-sectional study of 208 patients undergoing chemotherapy. A general characterization questionnaire and the Beck Depression Inventory were used. Results: most were women, between 40 and 79 years old, married, Catholic, retired/housewives, with elementary school and had no depression (71.2\%), while $17.3 \%$ had depression and $11.5 \%$ dysphoria. In the associations, it was found statistical significance in the associations of the Beck Depression Inventory with surgery $(p=0.002)$, time since surgery $(p=0.014)$, time since chemotherapy onset $(p=0.030)$, and side effects of chemotherapy $(p=0.019)$. Conclusion: there was a low incidence of depression, but the frequency was higher in patients who did not undergo concomitant surgery, who had started chemotherapy for up to six months and who reported more side effects during treatment.

Descriptors: Neoplasms; Depression; Drug Therapy; Oncology Nursing.

\footnotetext{
*Extraído do Trabalho de Conclusão de Curso "Presença de depressão em pacientes durante o tratamento oncológico quimioterápico", Universidade Federal do Triângulo Mineiro, 2018.

${ }^{1}$ Hospital de Amor de Barretos. Barretos, SP, Brasil.

${ }^{2}$ Universidade Federal do Triângulo Mineiro. Uberaba, MG, Brasil.

${ }^{3}$ Universidade Federal de Uberlândia. Uberlândia, MG, Brasil. 


\section{Introdução}

Devido ao crescimento mundial da população, o câncer tornou-se grave problema no contexto da saúde pública. A estimativa global, realizada no ano de 2018, pela International Agency for Research on Cancer, da Organização Mundial da Saúde, estimou 18,1 milhões de novas ocorrências de câncer, dos quais, aproximadamente, 60,0\% aconteceram em países em desenvolvimento. Para o ano de 2040, é previsto cerca de 29,5 milhões de novos casos, sendo que $80,0 \%$ destes acometerão países em desenvolvimento. No Brasil, prevê-se, ainda, 600 mil novas incidências de câncer em 2019 ${ }^{(1-2)}$.

Diante desse contexto, enfatiza-se que pacientes podem apresentar dificuldades no enfrentamento do diagnóstico e da terapia antineoplásica, além de incertezas, efeitos colaterais e complicações tardias decorrentes do tratamento, gerando sofrimento psicológico, como ansiedade e depressão ${ }^{(3-4)}$.

0 processo de mudança na vida de pacientes, em virtude da patologia decorrente de alterações psicoemocionais proporcionam o afastamento destes do convívio social e familiar, bem como profissional. A alteração emocional predispõe distorção da autoimagem e, consequentemente, ocorre redução da satisfação pessoal ${ }^{(5)}$.

Sentimentos negativos relacionados à doença e predisposições pessoais de alterações psicológicas têm aumentado o risco de pacientes com câncer desenvolverem ansiedade e depressão(6). Esta tem sido apontada como sintoma psicológico mais comum em pacientes com câncer, e a alta prevalência pode ser causada tanto pela doença quanto pelo tratamento realizado $^{(7)}$, principalmente pela quimioterapia ${ }^{(3)}$.

A carga de depressão pode influenciar a gravidade e o número de eventos adversos do tratamento realizado, aumentando outros sintomas decorrentes da terapia quimioterápica, como náusea e fadiga, comprometendo o desempenho de atividades cotidianas, do papel social, da adesão e continuidade ao tratamento, ocasionando diminuição da qualidade de vida de pacientes $^{(3,8-10)}$.

Nesse sentido, é fundamental que o enfermeiro, ao assistir o paciente durante a quimioterapia, possa identificar a presença de sintomas de depressão e, então, desenvolver estratégias ou intervenções para auxiliar o paciente no enfrentamento, com intuito de minimizar a presença destes sintomas e maximizar o bem-estar geral e a qualidade de vida.

Ao considerar que o diagnóstico e o tratamento do câncer estão aliados às emoções negativas, justifica-se o uso de instrumentos simples para investigar a ocorrência de sintomas de depressão nesses pacientes. Diante do exposto, a pesquisa objetivou analisar os fatores associados à depressão, em pacientes oncológicos, durante quimioterapia.

\section{Métodos}

Estudo descritivo, exploratório, de corte transversal, desenvolvido em hospital de ensino que realiza assistência a 27 municípios da macrorregião Triângulo Sul, do Estado de Minas Gerais, Brasil, sendo o único hospital que atende às pessoas em condições clínicas de alta complexidade, exclusivamente pelo Sistema Único de Saúde.

A amostragem utilizada foi proposital, sendo então selecionados os participantes considerados típicos da população pesquisada ${ }^{(11)}$, ou seja, nos dias em que os pesquisadores compareciam à central de quimioterapia para coleta de dados, abordavam os pacientes, convidando-os a participarem do estudo, mediante explicação do objetivo da pesquisa e aplicação dos critérios de inclusão e exclusão. Os participantes aprovados e que concordaram em participar foram inseridos na amostra, a qual foi constituída por 208 participantes.

Foram considerados aptos para pesquisa os participantes que atenderam aos critérios de inclu- 
são: ter idade igual ou superior a 18 anos, de ambos os sexos, com câncer e em tratamento quimioterápico na central de quimioterapia do hospital de ensino. Os pacientes que possuíam alguma dificuldade no entendimento de perguntas simples que pudesse comprometer as respostas dos questionários foram excluídos.

Os dados foram coletados entre maio de 2016 e janeiro de 2018, no dia em que os pacientes compareciam para realizar o tratamento, em enfermaria privativa ou coletiva, com leitos ou poltronas separadas por biombos. Três graduandas do curso de Enfermagem e coautoras deste estudo foram treinadas pelo professor orientador do projeto para padronização das entrevistas, que duravam cerca de 20 minutos e eram realizadas durante a sessão de quimioterapia.

As informações sociodemográficas e clínicas foram obtidas dos prontuários disponíveis e com o próprio paciente, através de questionário semiestruturado, construído pelo pesquisador principal e testado previamente para adequações, o qual abordou as variáveis: sexo, faixa etária, estado civil, profissão, cidade, nível de escolaridade, religião, tipo de câncer, esquema quimioterápico, tempo desde o início e presença de efeitos colaterais da quimioterapia, outros tratamentos, como radioterapia e cirurgia, tipo e tempo decorrido desde a cirurgia, se realizada.

O Inventário de Depressão de Beck (IDB) foi empregado para apreciar a presença de depressão. As versões deste são mundialmente utilizadas, tanto em amostras diagnosticadas quanto em não diagnosticadas clinicamente com depressão ${ }^{(12-13)}$, justificando-se o uso nesta pesquisa.

O IDB é considerado seguro e pertinente para avaliar a presença de sintomas depressivos na população não clínica, sendo composto por 21 itens relacionados a sensações de melancolia, pessimismo, derrota, insatisfação, remorso e de punição, descrédito, autoacusação, pensamentos suicidas, crises de pranto, irritabilidade, antissocialidade, dúvida, deturpação de imagem corporal, bloqueio para trabalho, sono irregular, fadiga, perda de apetite e peso, apreensão somática e redução de libido. Cada categoria é graduada com afirmações de zero a três, no qual o zero é considerado ausência e três a presença máxima do sintoma. 0 escore mínimo é zero e o máximo é 63. Para análise, o ponto de corte foi de até 15 para sem depressão; de 16 a 20 para detectar disforia; e de 21 a 63 para depressão, conforme diretrizes para amostras não diagnosticadas ${ }^{(12-13)}$.

Os dados obtidos foram organizados pelo software Microsoft Excel, versão 2016, por meio de dupla digitação. Posteriormente, esses dados foram transferidos para o software Statistical Package for the Social Sciences 23.0, para efetuar a análise estatística. Para análise descritiva da caracterização da amostra, calcularam-se as frequências e porcentagens. 0 Teste Exato de Fisher foi realizado para averiguar possíveis associações entre as variáveis, com limite de significância estatística 0,05.

A pesquisa foi submetida e aprovada pelo Comitê de Ética em Pesquisa da Universidade Federal do Triângulo Mineiro, conforme parecer $\mathrm{n}^{\mathrm{o}}$ 1.501.942/2016, de acordo com as recomendações da Resolução no 466/2012. Os participantes selecionados foram esclarecidos quanto aos objetivos da pesquisa e assinaram o Termo de Consentimento Livre e Esclarecido. Para confidencialidade, os questionários foram identificados por números sequenciais.

\section{Resultados}

Duzentos e oito pacientes participaram do estudo. Com relação aos aspectos sociodemográficos, predominaram o sexo feminino, $113(54,3 \%)$; na faixa etária entre 40 e 79 anos, 178(85,6\%); casados, 99(47,6\%); católicos, 128(61,5);aposentados, 52(25,0\%); e donas de casa 49(23,6\%); residentes na cidade sede da central de quimioterapia, $114(54,8 \%)$; e com nível de escolaridade o ensino fundamental, 126(60,6\%) (Tabela 1). 
Tabela 1 - Caracterização dos participantes quanto aos dados sociodemográficos $(\mathrm{n}=208)$

\begin{tabular}{|c|c|}
\hline Variáveis & n (\%) \\
\hline \multicolumn{2}{|l|}{ Sexo } \\
\hline Feminino & $113(54,3)$ \\
\hline Masculino & $95(45,7)$ \\
\hline \multicolumn{2}{|l|}{ Faixa etária (anos) } \\
\hline $18-39$ & $26(12,5)$ \\
\hline $40-79$ & $178(85,6)$ \\
\hline$\geq 80$ & $4(1,9)$ \\
\hline \multicolumn{2}{|l|}{ Estado civil } \\
\hline Casado & $99(47,6)$ \\
\hline Solteiro & $46(22,1)$ \\
\hline Viúvo & $32(15,4)$ \\
\hline Não informado & $31(14,9)$ \\
\hline \multicolumn{2}{|l|}{ Profissão/Ocupação } \\
\hline Aposentado & $52(25,0)$ \\
\hline Dona de casa & $49(23,6)$ \\
\hline Faxineira, diarista & $20(9,6)$ \\
\hline Comerciante, vendedor & $11(5,3)$ \\
\hline Outros & $76(36,5)$ \\
\hline \multicolumn{2}{|l|}{ Cidade/Procedência } \\
\hline Uberaba & $114(54,8)$ \\
\hline $\begin{array}{l}\text { Cidades da macrorregião Triângulo Sul de Minas } \\
\text { Gerais }\end{array}$ & $75(36,1)$ \\
\hline Outas cidades & $19(9,1)$ \\
\hline \multicolumn{2}{|l|}{ Nível de escolaridade } \\
\hline Ensino Fundamental & $126(60,6)$ \\
\hline Ensino Médio & $67(32,2)$ \\
\hline Ensino Superior & $15(7,2)$ \\
\hline \multicolumn{2}{|l|}{ Religião } \\
\hline Católico & $128(61,5)$ \\
\hline Protestante & $33(15,9)$ \\
\hline Espírita & $27(13,0)$ \\
\hline Sem religião definida/Não informado & $20(9,6)$ \\
\hline
\end{tabular}

Quanto aos dados clínicos, os tipos de cânceres mais encontrados foram: cólon e reto, em 38 pacientes (18,3\%); mama, em 21 (10,1\%); colo de útero e pulmão, em 13 (6,3\%) cada; estômago, em 12 (5,8\%); linfoma de Hodgkin, em 10 (4,8\%); leucemia, em nove (4,3\%); linfoma não Hodgkin, em sete $(3,4 \%)$; próstata, em seis $(2,9 \%)$; esôfago, em três $(1,4 \%)$; e outros tipos, com um a dois pacientes para cada câncer.

Com relação a outros tratamentos realizados, mais da metade dos pacientes realizaram cirurgia e menos de um terço, radioterapia (Tabela 2).
Tabela 2 - Caracterização dos participantes quanto aos dados clínicos (n=208)

\begin{tabular}{lc}
\hline Variáveis & $\mathbf{( \% )}$ \\
\hline Tipo de cirurgia & \\
Não realizou & $102(49,0)$ \\
Retirada parcial do órgão afetado & $33(15,9)$ \\
Retirada de tumor, nódulos e/ou adjacências & $27(13,0)$ \\
Biópsia & $21(10,1)$ \\
Retira total do órgão afetado & $14(6,7)$ \\
Transplante & $6(2,9)$ \\
Não soube informar & $5(2,4)$ \\
Tempo decorrido desde a cirurgia (meses) & \\
Não realizaram & $102(49,0)$ \\
$\leq 3$ & $33(15,9)$ \\
$>3$ & $73(35,1)$ \\
Realização de radioterapia & \\
Sim & $63(30,3)$ \\
Não & $145(69,7)$ \\
Tempo decorrido desde o início da quimioterapia (meses) & $76(36,5)$ \\
$\leq 1$ & $81(39,0)$ \\
26 & $51(24,5)$ \\
\hline &
\end{tabular}

Em relação à quimioterapia, foram encontrados 59 esquemas diferentes em 135 sujeitos. Houve perda de registro do esquema quimioterápico em 73 sujeitos, devido a não acessibilidade à prescrição médica no prontuário, no momento da entrevista. Destes esquemas, os mais utilizados foram: Fluorouracila associado à Leucovorine e Oxaliplatina, utilizado em 26 $(19,3 \%)$ pacientes; Cisplatina, em nove $(6,7 \%)$ pacientes; Fluorouracila, em oito $(5,9 \%)$ pacientes; Pamidronato, em sete $(5,2 \%)$ pacientes; Paclitaxel associado à Carboplatina, em sete $(5,2 \%)$ indivíduos.

Sobre os efeitos colaterais da quimioterapia, $138(66,4 \%)$ pacientes referiram sintomas e 70 $(33,7 \%)$ estavam assintomáticos. Dentre os relatos, 78 $(37,5 \%)$ pacientes apresentaram sintomas gastrointestinais (náuseas, vômitos ou diarreia) associados a sintomas físicos (fadiga, sudorese, fraqueza, sonolência, insônia ou prostração), 30 (14,4\%) indivíduos referiram sintomatologia gastrointestinal, 28 (13,5\%) 
pacientes identificaram sintomas físicos, um $(0,5 \%)$ relatou efeitos colaterais emocionais (ansiedade, angústia, irritabilidade ou preocupação) e um $(0,5 \%)$ apresentou sintomas gastrointestinais associados a sintomas físicos e emocionais.

De acordo com o IDB, para amostras não diagnosticadas clinicamente, a média dos escores indicou que a maioria, $148(71,2 \%)$ pacientes, encontrava-se sem depressão no momento da entrevista. Contudo, $36(17,3 \%)$ indivíduos apresentavam depressão e 24 $(11,5 \%)$ disforia.
Na realização de associações das variáveis sociodemográficas com os escores do IDB, não foram encontradas associações significativas. No entanto, notou-se que, entre os sexos, o dobro de mulheres apresentou depressão ( $\mathrm{p}=0,200)$.

Com as variáveis clínicas, houve associações estatisticamente significativas $(\mathrm{p}<0,05)$ do IDB com as variáveis: realização de cirurgia, tempo decorrido desde a cirurgia, tempo de início da quimioterapia (em meses) e efeitos colaterais da quimioterapia (Tabela 3).

Tabela 3 - Associação entre escores do IDB com variáveis clínicas (n=208)

\begin{tabular}{|c|c|c|c|c|c|}
\hline \multirow[t]{2}{*}{ Variáveis clínicas } & \multirow{2}{*}{$\begin{array}{c}\begin{array}{c}\text { Escores sem } \\
\text { depressão }\end{array} \\
\text { n (148) }\end{array}$} & \multirow{2}{*}{$\begin{array}{c}\begin{array}{c}\text { Escores de } \\
\text { disforia }\end{array} \\
\mathbf{n}(24)\end{array}$} & \multirow{2}{*}{$\begin{array}{c}\begin{array}{c}\text { Escores de } \\
\text { depressão }\end{array} \\
\mathbf{n}(36)\end{array}$} & \multirow{2}{*}{$\begin{array}{c}\text { Total } \\
\text { n (208) }\end{array}$} & \multirow[t]{2}{*}{$\mathbf{p}^{*}$} \\
\hline & & & & & \\
\hline \multicolumn{6}{|l|}{ Realização de cirurgia } \\
\hline $\operatorname{Sim}$ & 84 & 13 & 9 & 106 & 0,002 \\
\hline Não & 64 & 11 & 27 & 102 & \\
\hline \multicolumn{6}{|l|}{ Tempo decorrido desde a cirurgia (meses) } \\
\hline$\leq 3$ & 28 & 3 & 2 & 33 & \\
\hline$>3$ & 56 & 10 & 7 & 73 & \\
\hline \multicolumn{6}{|c|}{ Tempo decorrido desde o início da quimioterapia (meses) } \\
\hline$\leq 1$ & 62 & 3 & 11 & 76 & 0,030 \\
\hline $1-6$ & 51 & 12 & 18 & 81 & \\
\hline$>6$ & 35 & 9 & 7 & 51 & \\
\hline Assintomáticos & 57 & 6 & 7 & 70 & 0,019 \\
\hline Gastrintestinais e físicos & 52 & 10 & 16 & 78 & \\
\hline Gastrintestinais & 19 & 2 & 9 & 30 & \\
\hline Físicos & 20 & 6 & 2 & 28 & \\
\hline Emocionais & 0 & 0 & 1 & 1 & \\
\hline Gastrointestinais, físicos e emocionais & 0 & 0 & 1 & 1 & \\
\hline
\end{tabular}

\section{Discussão}

O estudo apresentou limitações que se referem ao tipo de estudo, à coleta de dados e amostra. Este estudo de corte transversal não possibilitou avaliar as mudanças ocorridas nos sintomas de depressão no decorrer do tratamento. Em relação à coleta de dados, houve entrada de acompanhantes e/ou profissionais assistenciais na enfermaria, o que poderia influenciar a concentração de participantes no momento da resposta, sendo interrompida a entrevista e retomada somente após saída daqueles. Quanto à amostra, em virtude da diversidade de tipos de câncer e protocolos quimioterápicos encontrados.

Acredita-se que estes resultados podem contribuir para enfermeiros que atuam na Oncologia, na identificação precoce da presença de sintomas de depressão nestes pacientes, para então planejar assistência holística, propor intervenções para melhorá-los, e se julgar necessário, encaminhar para outros profissionais de saúde, como médico ou psicólogo, para que, em conjunto, possam cuidar do paciente oncológico.

A presente pesquisa teve a caracterização da 
amostra semelhante a outra investigação realizada com 15 participantes que frequentavam sociedade de apoio ao câncer, com predominância de mulheres, na faixa etária entre 40 e 60 anos, católicas e com nível escolar baixo, e divergiu em relação ao tipo de neoplasia encontrada que foi, em ordem decrescente, o câncer de mama, de ovário e pulmão(5).

Outro trabalho brasileiro, desenvolvido com 96 idosos com câncer, apresentou 50,0\% de mulheres na amostra. A maioria dos entrevistados era casada, com baixo nível de escolaridade (até quatro anos de estudo), baixa renda (menos que um salário mínimo), com tumor em estágio avançado e realizando quimioterapia; como tratamento prévio, 51,0\% realizaram a cirurgia e $35,4 \%$, a radioterapia ${ }^{(9)}$, corroborando com este estudo.

Enfatiza-se variável significante quanto à presença de sintomas de depressão, devido às diferentes abordagens (pacientes diagnosticados ${ }^{(6,14)}$ ou não clinicamente $^{(4,8-9,15)}$ e instrumentos utilizados, como IDB $^{(3,5,8,16-17)}$, Center for Epidemiologic Studies Depression Scale ${ }^{((7)}$, Escala de Depressão Geriátrica ${ }^{(9)}$, Escala Hospitalar de Ansiedade e Depressão ${ }^{(4,14-19)}$, Self-rating Depression Scale ${ }^{(20)}$.

Estudo com idosos com câncer deparou com $17,7 \%$ apresentando sintomas de depressão( ${ }^{(9)}$, apoiando este trabalho, porém divergindo da pesquisa que avaliou 63 pacientes com câncer de mama após quimioterapia, em que encontraram $46,0 \%$ de pacientes com depressão ${ }^{(20)}$.

Estudo retrospectivo, realizado em Taiwan com 36.586 mulheres com câncer de mama que receberam tratamento adjuvante, no período de seis anos, analisou o risco subsequente de transtornos depressivos, encontrando taxa de incidência de 5,3\% de distúrbios depressivos diagnosticados clinicamente, cujo risco destes transtornos foi maior quando as pacientes tinham entre 40 e 59 anos de idade e receberam terapias adjuvantes, incluindo quimioterapia e radioterapia $^{(6)}$.

Ainda em relação à depressão, pacientes em tratamento que possuíam ansiedade também apre- sentavam maior intensidade para depressão. Ambos os transtornos podem ser provenientes do diagnóstico médico, do método terapêutico utilizado e da realização ou não de procedimento cirúrgico ${ }^{(16)}$. No presente estudo, não foi possível correlacionar os dois distúrbios.

Na comparação entre os sexos, estudos ${ }^{(4,9,14,18)}$ encontraram que mulheres apresentaram escores maiores de depressão em relação aos homens, indo ao encontro deste estudo, porém sem significância estatística.

Diante disso, é possível observar que o tratamento para o câncer tem causado manifestação de sintomas de depressão e ansiedade em pacientes e que fatores, como idade, sexo, tipo de câncer, outras terapias e tempo de tratamento podem influenciar na intensidade dos sintomas.

Investigação realizada em chineses com câncer e respectivos cuidadores encontrou que quase um terço dos participantes relatou ansiedade e depressão. Nos adultos com câncer entrevistados, vários fatores influenciaram a presença de ansiedade e depressão, como idade, sexo, estado civil, ser informado sobre a doença, diferentes tipos de câncer e tratamento ${ }^{(15)}$.

Em estudo realizado com 3.370 sobreviventes de câncer na Alemanha, fatores como estar desempregado, receber pouco apoio social, estar sem tratamento, menor tempo desde o diagnóstico, saúde física prejudicada e pertencer à família de baixa renda obtiveram associações estatisticamente significantes para presença de maiores escores de depressão. Apuraram que mulheres ou homens com câncer de órgão genital ou câncer hematológico tiveram associações estatisticamente significantes a escores mais altos de depressão, em comparação com sobreviventes com câncer de $\operatorname{mama}^{(19)}$.

Estudo de Taiwan encontrou que pacientes com câncer colorretal em tratamento obtiveram maiores escores para depressão e fadiga do que aqueles que finalizaram o tratamento ${ }^{(7)}$.

Pesquisa brasileira analisou os fatores relacionados aos sintomas indicativos de depressão em 
mulheres com câncer de mama e encontrou que idade, escolaridade, diagnóstico de outras doenças, tipo de cirurgia, linfedema, autoestima e imagem corporal foram fatores associados à sintomologia depressiva, mesmo depois da cura da malignidade ${ }^{(8)}$.

Com o decorrer do tempo e do tratamento quimioterápico, a gravidade prévia dos sintomas de depressão, fadiga e distúrbios do sono previu a gravidade subsequente do mesmo sintoma em dois grupos de mulheres (pré e pós-menopausa). Ao relacionar os sintomas, a fadiga, no início anteviu a depressão, no decorrer do tratamento, em mulheres mais jovens no grupo pré-menopausa ${ }^{(17)}$.

Estudo realizado na Croácia em 147 mulheres com câncer de mama encontrou que a fadiga foi relacionada positivamente, enquanto que a idade e o tratamento diário no hospital foram negativamente associados à depressão medida pelo IDB(3).

Observa-se que o tratamento quimioterápico ocasionou alterações em pacientes, acarretando modificações nos hábitos de vida. Tais mudanças interferem no estado psicológico, promovendo sentimentos de tristeza, medo e angústia, e, consequentemente, refletem a sintomatologia depressiva presente no cotidiano desses pacientes.

\section{Conclusão}

Houve baixa incidência de depressão, contudo a frequência foi maior nos participantes que não realizaram cirurgia concomitante, que tiveram quimioterapia iniciada até seis meses e que relataram mais efeitos colaterais durante o tratamento.

\section{Colaborações}

Arantes TC contribuiu com a concepção do projeto, interpretação e organização dos dados. Martins VE e Mendes AS colaboraram com a concepção do projeto. Silva AMB contribuiu com a revisão crítica relevante do conteúdo intelectual. Nicolussi AC colaborou na concepção do projeto, análise e interpretação dos dados, revisão crítica e aprovação final da versão a ser publicada.

\section{Referências}

1. World Health Organization. International Agency for Research on Cancer. Globocan 2018 [Internet]. 2018 [cited July 15, 2019]. Available from: http:// globocan.iarc.fr/tomorrow/home

2. Ministério da Saúde (BR). Instituto Nacional de Câncer José Alencar Gomes da Silva (INCA). Coordenação de Prevenção e Vigilância. Estimativa 2018: incidência de câncer no Brasil [Internet]. 2017 [citado 2019 Jul 15]. Disponível em: https:// www.inca.gov.br/publicacoes/livros/estimativa-2018-incidencia-de-cancer-no-brasil

3. Separovic R, Silovski T, Vuger AT, Bajic Z, Silovski $\mathrm{H}$, Juric A. Association of breast cancer symptoms with patients' quality of life and depression; a Croatian cross-sectional study. Psychiatr Danub [Internet]. 2019 [cited July 23, 2019]; 31(Suppl. 1):92-8. Available from: http://www.psychiatriadanubina.com/UserDocsImages/pdf/dnb_vol31_ sup1/dnb_vol31_sup1_92.pdf

4. Hovaldt HB, Andersen I, Sandager M, Sperling C, Frederiksen K, Bidstrup PE, et al. The role of social position and anxiety and depressive symptoms among Danish cancer survivors. Acta Oncol. 2015; 54(5):693-703. doi: https://doi.org/10.3109/0284186X.2014.996666

5. Miranda SL, Lanna MAL, Felippe WC. Espiritualidade, depressão e qualidade de vida no enfrentamento do câncer: estudo exploratório. Psicol Cienc Prof. 2015; 35(3):870-85. doi: http://dx. doi.org/10.1590/1982-3703002342013

6. Chang $\mathrm{CH}$, Chen SJ, Liu CY. Adjuvant treatments of breast cancer increase the risk of depressive disorders: a population-based study. J Affect Disorders. 2015; 182:44-9. doi: https://doi.org/10.1016/j. jad.2015.04.027

7. Tung HY, Chao TB, Lin YH, Wu SF, Lee HY, Ching CY, et al. Depression, fatigue, and QoL in colorectal cancer patients during and after treatment. West J Nurs Res. 2016; 38(7):893-908. http://dx.doi. org/10.1177/0193945916630256 
8. Boing L, Pereira GS, Araújo CCR, Sperandio FF, Loch MSG, Bergmann A, et al. Factors associated with depression symptoms in women after breast cancer. Rev Saúde Pública. 2019; 53:30. doi: https:// doi.org/10.11606/S1518-8787.2019053000786

9. Santos CA, Ribeiro AQ, Rosa COB, Ribeiro RCL. Depressão, déficit cognitivo e fatores associados à desnutrição em idosos com câncer. Cienc Saúde Coletiva. 2015; 20(3):751-60. doi: http://dx.doi. org/10.1590/1413-81232015203.06252014

10. Hamish RS. Depression in cancer patients: pathogenesis, implications and treatment. Oncol Lett. 2015; 9(4):1509-14. doi: http://dx.doi. org/10.3892/ol.2015.2944

11. Polit DF, Beck CT. Fundamentos de pesquisa em enfermagem: avaliação de evidências para a prática da enfermagem. Porto Alegre: Artmed; 2019.

12. Gorestein C, Andrade L. Inventário de depressão de Beck: propriedades psicométricas da versão em português. Rev Psiquiatr Clin [Internet]. 1998 [citado 2019 Jul 15]; 25(5):245-50. Disponível em:https://www.researchgate.net/publication/284700806_Inventario_de_depressao_de Beck_Propriedades_psicometricas_da_versao_ em_portugues

13. Gomes-Oliveira MH, Gorestein C, Lotufo Neto F, Andrade LH, Wang YP. Validation of the Brazilian Portuguese version of the Beck Depression Inventory-II in a community sample. Rev Bras Psiquiatr. 2012; 34(4):389-94. doi: http://dx.doi. org/10.1016/j.rbp.2012.03.005

14. Lima MP, Oliveira DS, Irigaray TQ. Symptoms of depression and anxiety in cancer outpatients: predictive variables. Psicooncol. 2018; 15(2):37384. doi: http://dx.doi.org/10.5209/PSIC.61443
15. Li Q, Lin Y, Xu Y, Zhou H. The impact of depression and anxiety on quality of life in Chinese cancer patient-family caregiver dyads, a cross-sectional study. Health Qual Life Outcomes. 2018; 16(1):230. doi: https://doi.org/10.1186/s12955018-1051-3

16. Kaminska M, Kubiatowski T, Ciszewski T, Czarnocki KJ, Makara-Studzinska M, Bojar I, et al. Evolution of symptoms of anxiety and depression in women with breast cancer after breast amputation or conservation treated with adjuvant chemotherapy. Ann Agric Environ Med. 2015; 22(1):185-9. doi: https://doi.org/10.5604/12321966.1141392

17. Ho SY, Rohan KJ, Parent J, Tager FA, McKinley PS. A longitudinal study of depression, fatigue, and sleep disturbances as a symptom cluster in women with breast cancer. J Pain Symptom Manage. 2015; 49(4):707-15. doi: http://dx.doi.org/10.1016/j. jpainsymman.2014.09.009

18. Bergerot CD, Mitchell HR, Ashing KT, Kim Y. A prospective study of changes in anxiety, depression, and problems in living during chemotherapy treatments: effects of age and gender. Support Care Cancer. 2017; 25(6):1897-904. doi: https:// doi.org/10.1007/s00520-017-3596-9

19. Inhestern L, Beierlein V, Bultmann JC, Möller B, Romer G, Koch U, et al. Anxiety and depression in working-age cancer survivors: a register-based study. BMC Cancer. 2017; 17(1):347. doi: https:// doi.org/10.1186/s12885-017-3347-9

20. Huang Z, Zhao J, Ding K, Lv Y, Zhang C, Chao H, et al. Depression involved in self-reported prospective memory problems in survivors of breast cancer who have received chemotherapy. Medicine. 2019; 98(16):e15301. doi: https://doi.org/10.1097/ MD.0000000000015301 